\title{
Enhanced Heat Transfer with Metal-Dielectric Core-Shell Nanoparticles
}

\author{
Ali Alkurdi® ${ }^{1, \dagger}$ Julien Lombard $\odot,{ }^{1,2, \dagger}$ François Detcheverry $\odot,{ }^{1}$ and Samy Merabia ${ }^{1,{ }^{*}}$ \\ ${ }^{1}$ Université de Lyon, Université Claude Bernard Lyon 1, CNRS, Institut Lumière Matière, \\ 69622 Villeurbanne, France \\ ${ }^{2}$ Departamento de Física y Química Teórica, Facultad de Química, Universidad Nacional Autónoma de Mexico, \\ Mexico City 04510, Mexico
}

(Received 27 August 2019; revised manuscript received 22 November 2019; accepted 24 January 2020; published 13 March 2020)

\begin{abstract}
Heat transfer from irradiated metallic nanoparticles is relevant to a broad array of applications ranging from water desalination to photoacoustics. The efficiency of such processes depends on the ability of these nanoparticles to absorb the pulsed illuminating light and to quickly transfer energy to the environment. Here we show that compared with homogeneous gold nanoparticles having the same size, gold-silica core-shell nanoparticles enable heat transfer to liquid water that is faster. We reach this conclusion by considering both analytical and numerical calculations. The key factor explaining enhanced heat transfer is the direct interfacial coupling between metal electrons and silica phonons. We discuss how to achieve fast heating of water in the vicinity of the particle and show that optimal conditions involve nanoparticles with thin silica shells irradiated by ultrafast laser pulses. Our findings should serve as guides for the optimization of thermoplasmonic applications of core-shell nanoparticles.
\end{abstract}

DOI: 10.1103/PhysRevApplied.13.034036

\section{INTRODUCTION}

Metallic nanoparticles have a remarkable ability to absorb electromagnetic radiation in the vicinity of the plasmon resonance wavelength, which lies in the visible or near-infrared domain for common nanostructures [1]. This phenomenon originates in the collective electromagnetic response of the metal conduction electrons, which results in local field enhancement. In turn, this localized enhancement may be exploited to realize heat sources at the nanoscale [2-4]. By tailoring of the nanoparticle size and composition, the resonance wavelength may be tuned, which is advantageous if electromagnetic absorption or scattering from the nanoparticle environment has to be minimized.

This sensitivity combined with the biocompatibility of gold nanoparticles (GNPs) has been exploited in different contexts, including thermally induced photochemistry [5], phase transition [6], material growth [7], and nanomedicine $[8,9]$. The latter includes thermal imaging [10], diagnosis, photoacoustics [11], drug release $[12,13]$, and cancer therapy $[14,15]$, in which GNPs have been used to induce localized heating in tumor cells, leading to their destruction. In these applications, the efficiency of the treatment depends on the absorption contrast between the cancerous and healthy tissues [4], which is

\footnotetext{
*smerabia@gmail.com

†These authors contributed equally to this work.
}

realized by working in the so-called transparency window between 700 and $900 \mathrm{~nm}$, where absorption by human tissues is a minimum [16].

Alongside GNPs, metal-dielectric core-shell nanoparticles (CSNPs) have also been investigated as a nanosource of heat with applications in cancer therapy [17], optical enhancement [18,19], and solar-energy-assisted water purification [20], bringing in some cases clear benefits. One such instance is photoacoustics, where the rapid heating of illuminated particles triggers an acoustic or stress wave induced by thermal expansion [11]. The efficiency of this process is intimately related to the ability of the nanoparticles to transfer heat in their environment. Chen et al. [10,21] used gold nanorods coated with silica to assess the ability of these nano-objects to enhance the contrast in photoacoustic imaging. They found that compared with bare gold nanorods, silica-coated nanorods produce photoacoustic signals that are about 3 times higher. This enhancement is shown not to result from changes in the absorption cross section due to the silica coating; rather it is ascribed to a change in interfacial thermal conductivity induced by the silica shell. This interpretation is consistent with earlier experimental investigations of $\mathrm{Hu}$ et al. [22], who reported faster heat dissipation for gold nanoparticles coated with a silica shell. The underlying mechanism, however, has not been elucidated so far.

In a different context, several experimental studies investigated heat transport at metal-dielectric interfaces, on picosecond timescales. Recent transient-thermoreflectance 
experiments with a gold film on silicon and silica substrates [23,24] revealed the existence of a direct electron-substrate energy transfer. Indeed, there are at least two mechanisms according to which electrons may heat up the substrate: nonequilibrium electron-phonon exchange in the metal, which then transfers energy to the substrate through phonon-phonon coupling [25-29]; and direct electron-phonon coupling through the interface [30-33]. While the effect of the electron-phonon coupling in the bulk metal is to increase the thermal boundary conductance $[25,34]$, the direct electron-phonon coupling provides another channel for heat to flow across the metaldielectric interface [35].

This energy transfer through interfacial electron-phonon conductance was predicted theoretically 20 years ago [30-33]. Although the exact nature of the coupling between the conduction electrons and the substrate phonons remains under investigation [36], experiments report conductance values in the range from 100 to 1000 $\mathrm{MW} / \mathrm{m}^{2} / \mathrm{K}[23,24]$, in agreement with theoretical predictions. One consequence recently emphasized by Lombard et al. [35] is that thermal transport at metal-nonmetal interfaces is enhanced when such electron-phonon conductance is present.

Whereas several theoretical and experimental studies have characterized heat generation under pulsed illumination of GNPs [3,6,37-39], the case of CSNPs has received much less attention so far. Exceptions include Refs. [18,40], which are, however, restricted to static conditions only. Recent computational studies also investigated the photoacoustic response of silica-coated gold nanospheres $[41,42]$. However, these studies consider only one temperature to describe heat transfer in the coreshell nanoparticle, an approximation that is reasonable for nanosecond laser pulses, but is insufficient for femtosecond and picosecond pulses. Under these conditions, as we will show, electron-phonon processes play the leading role in transferring heat to the nanoparticle environment.

In this work, we study theoretically transient heat transfer around gold-silica CSNPs illuminated by a pulsed laser. We consider a multitemperature model, in which each energy carrier is assigned a temperature field. Our calculations demonstrate that, at a constant value of the laser power, core-shell nanoparticles may heat up the surrounding water faster than gold nanoparticles of the same diameter. This effect is significant for a thin silica shell and very short laser-pulse durations. Of prime importance is the role of the interfacial electron substrate conductance, which permits rapid heating of the silica shell, eventually accelerating heat transfer to water. The remainder of this article is organized as follows. Section II introduces the model, for which an analytical approach is developed in Sec. III. Numerical results are presented and discussed in Sec. IV. A summary and perspectives are given in Sec. V.

\section{MODEL}

Our model is akin to a two-temperature model (TTM). We first present the governing equations before discussing in detail the underlying assumptions and range of validity of this approach.

\section{A. Governing equations}

The system we investigate is a core-shell nanoparticle, with metallic core and nonmetallic shell [Fig. 1(a)]. Although our description is general, we focus in particular on the gold-silica combination, which has been investigated experimentally [10]. The nanoparticle is immersed in water and submitted to laser pulsed illumination. Figure 1(b) recapitulates the various energy fluxes at play during the relaxation process. The light energy is instantaneously absorbed by the free electrons in the metal, causing a rapid increase in their temperature. This excess energy is then transferred to the shell by two different channels.
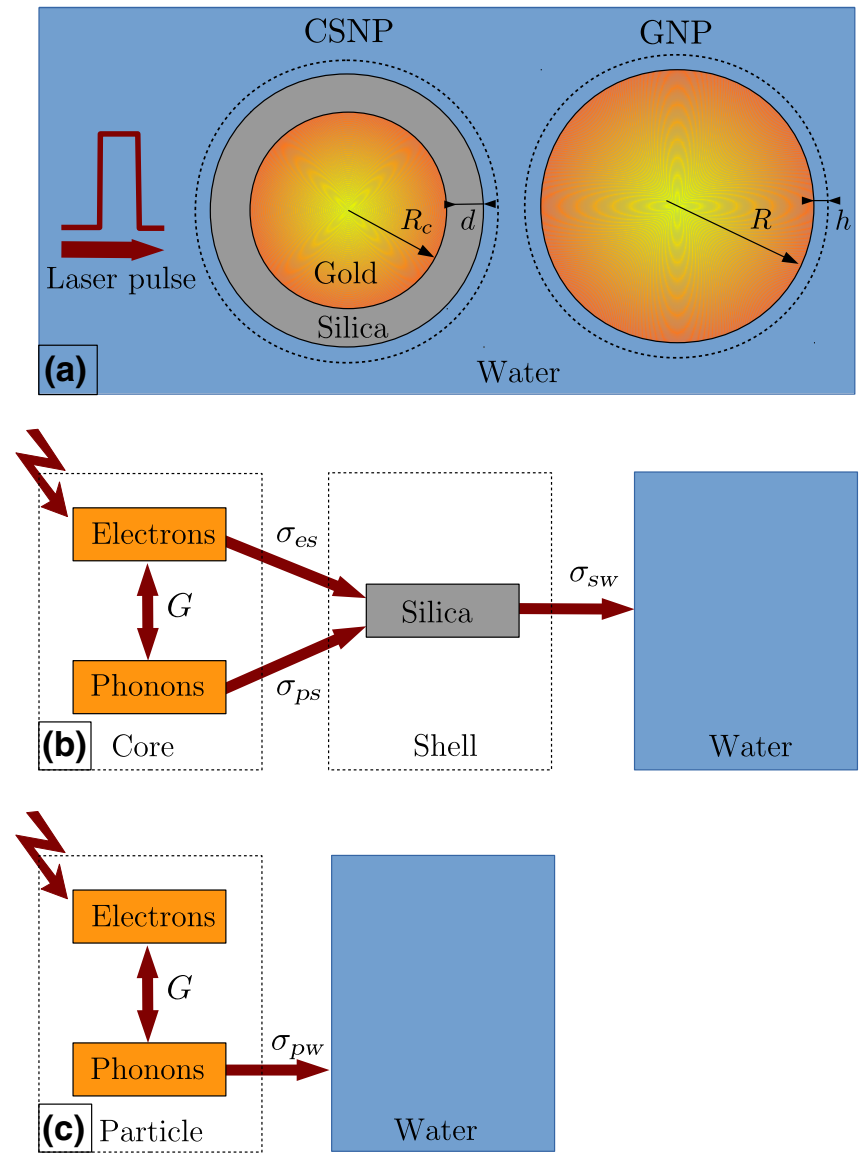

FIG. 1. Heat transfer from a CSNP and a GNP heated by a laser pulse. $G$ is the coupling constant between electrons and phonons in the metal. $\sigma_{e s}, \sigma_{p s}, \sigma_{s w}$, and $\sigma_{p w}$ are the interfacial conductances at the electron-silica, phonon-silica, silica-water, and phonon-water interfaces, respectively. 
The first is direct and occurs at the core-shell interface through the electron-phonon thermal conductance $\sigma_{e s}$. The second channel is indirect: it involves electron-phonon energy exchange inside the core, as governed by the coupling factor $G$, and then phonon-phonon thermal coupling at the core-shell interface, quantified by the conductance $\sigma_{p s}$. With the heat diffusing in the shell, a thermal flux proportional to the phonon-water conductance $\sigma_{s w}$ is induced across the shell-water interface, allowing the energy to diffuse in the infinite water reservoir. Although our focus is on a CSNP, we use throughout the study the bare gold nanoparticle as a reference point. The heat-transfer channels relevant in this case are depicted in Fig. 1(c): while electrons and phonons thermally equilibrate in the metal, a thermal flux is induced across the metalwater interface through the interfacial phonon-phonon conductance $\sigma_{p w}$.

The thermal processes are assumed to be governed by the following set of equations:

$$
\begin{aligned}
V_{c} c_{e} \frac{\partial T_{e}(t)}{\partial t}= & -V_{c} G\left(T_{e}-T_{p}\right) \\
& -S_{c} \sigma_{e s}\left[T_{e}-T_{s}\left(R_{c}, t\right)\right]+\mathcal{P}(t), \\
V_{c} c_{p} \frac{\partial T_{p}(t)}{\partial t}= & V_{c} G\left(T_{e}-T_{p}\right) \\
& -S_{c} \sigma_{p s}\left[T_{p}-T_{s}\left(R_{c}, t\right)\right] \\
c_{s} \frac{\partial T_{s}(r, t)}{\partial t}= & k_{s} \nabla^{2} T_{s} \\
c_{w} \frac{\partial T_{w}(r, t)}{\partial t}= & k_{w} \nabla^{2} T_{w}
\end{aligned}
$$

where the index $m \in\{e, p, s, w\}$ indicates the component [43] and $T_{m}, c_{m}$, and $k_{m}$ are, respectively, the temperature, heat capacity, and thermal conductivity of component $m$, the thermal boundary conductance at the interface between component $m$ and component $n$ is indicated by $\sigma_{m n}, r$ is the radial distance, $R_{c}, S_{c}$, and $V_{c}$ are the radius, surface area, and volume of the core domain, and $R=R_{c}+d, S$, and $V$ are the same quantities for the entire nanoparticle, including the shell of thickness $d$, whose volume $V_{s}=V-V_{c}$. For the power $\mathcal{P}(t)$ received by the electrons, we consider for simplicity a square pulse $\mathcal{P}(t)=\bar{P} \Theta\left(t_{\text {pul }}-t\right)$, with $\bar{P}$ the average laser power, $\Theta$ the Heaviside function, and $t_{\text {pul }}$ the pulse duration. Note that the average power $\bar{P}$ is related to the laser fluence $F$ and nanoparticle absorption cross section $\sigma_{\text {abs }}(\lambda)$ simply through $\bar{P}=\sigma_{\text {abs }}(\lambda) F / t_{\text {pul }}$, where $\sigma_{\mathrm{abs}}$ may be described by Mie theory $[1,3]$ for the spherical nanoparticles of interest here.

In addition to Eqs. (1)-(4) describing the temporal evolution of temperatures, the continuity of the heat flux across an interface implies the following boundary condi-
TABLE I. Thermophysical parameters at $T_{0}=300 \mathrm{~K}$ for the components of the system considered: thermal conductivity $k$, heat capacity $c$, and diffusivity $\mathcal{D}=k / c$.

\begin{tabular}{lccccc}
\hline \hline Quantity & Electron & Phonon & Silica & Water & Unit \\
\hline$k$ & 320.0 & 3.0 & 1.37 & 0.606 & $\mathrm{~W} \mathrm{~m}^{-1} \mathrm{~K}^{-1}$ \\
$c$ & 0.0197 & 2.35 & 1.01 & 4.11 & $\mathrm{MJ} \mathrm{m}^{-3} \mathrm{~K}^{-1}$ \\
$\mathcal{D}$ & 16243 & 1.28 & 1.36 & 0.15 & $\mathrm{~nm}^{2} \mathrm{ps}^{-1}$ \\
\hline \hline
\end{tabular}

tions at the core-shell interface $r=R_{c}$ and the shell-water interface $r=R$ :

$$
\begin{aligned}
-k_{s} \nabla T_{s}\left(R_{c}, t\right)= & \sigma_{e s}\left[T_{e}-T_{s}\left(R_{c}, t\right)\right] \\
& +\sigma_{p s}\left[T_{p}-T_{s}\left(R_{c}, t\right)\right], \\
-k_{s} \nabla T_{s}(R, t)= & \sigma_{s w}\left[T_{s}(R, t)-T_{w}(R, t)\right], \\
= & -k_{w} \nabla T_{w}(R, t) .
\end{aligned}
$$

Finally, as regards initial conditions, all components are assumed to be at room temperature $T_{0}$. The equations for the GNP can be written in a similar manner. For completeness, they are given in Supplemental Material [44].

We list in Tables I and II the parameters relevant for gold-silica CSNPs. Two comments are in order on the value of the interfacial conductances. First, the thermal conductance between the gold nanoparticle and water depends on the wetting contact angle, and increases with the hydrophilicity of the interface, as discussed in [45-47]. Here, for the evaluation of $\sigma_{s w}$, we consider the nanoparticle-water interface to be hydrophilic. This choice is justified by the fact that hydrophilic nanoparticlewater interfaces are preferred experimentally to ensure good dispersion of the nanoparticles and avoid nanoparticle agglomeration, which may occur if the interface is hydrophobic. Second, the interfacial electron-phonon coupling is assumed to be local, while it has been proposed that this coupling may extend a few angstroms in the dielectric $[30,36]$. These latter studies demonstrate that the effect of this spatial extension is to slightly decrease the total thermal boundary resistance [36]. Therefore, taking into account these nonlocal effects would only tend to accelerate the heating of liquid water. However, since the exact spatial coupling length in silica is not known, we work

TABLE II. Thermal exchange coefficients in bulk gold and at the interfaces between the various components.

\begin{tabular}{lccc}
\hline \hline Quantity & Value & Unit & References \\
\hline$G$ & $2.5 \times 10^{10}$ & $\mathrm{MW} \mathrm{m}^{-3} \mathrm{~K}^{-1}$ & {$[49]$} \\
$\sigma_{e s}\left(T_{0}\right)$ & 152 & $\mathrm{MW} \mathrm{m}^{-2} \mathrm{~K}^{-1}$ & {$[23]$} \\
$\sigma_{e s}\left(10 T_{0}\right)$ & 636 & $\ldots$ & {$[23]$} \\
$\sigma_{p s}$ & 141 & $\ldots$ & {$[35]$} \\
$\sigma_{s w}$ & 150 & $\cdots$ & {$[50]$} \\
$\sigma_{p w}$ & 139 & $\cdots$ & {$[45,46,51]$} \\
\hline \hline
\end{tabular}


with a local electron-phonon coupling. If not otherwise mentioned, all properties are taken at $T_{0}=300 \mathrm{~K}$. There are two quantities, however, for which it is essential that the temperature dependence is taken into account. The first is the electronic heat capacity $c_{e}=\gamma T_{e}$, with $\gamma$ the Sommerfeld constant of the metal [48]. For gold, $\gamma=$ $65.6 \mathrm{~J} \mathrm{~m}^{-3} \mathrm{~K}^{-2}$ [23]. The second is the interfacial electronphonon conductance at the core-shell interface, which can be written as

$$
\sigma_{e s}\left(T_{e}\right)=A+B T_{e}
$$

with the constants $A=96.1 \mathrm{MW} \mathrm{m}^{-2} \mathrm{~K}^{-1}$ and $B=$ $0.18 \mathrm{MW} \mathrm{m}^{-2} \mathrm{~K}^{-2}$ [23].

\section{B. Discussion}

A complete treatment of the heat transfers in our system should be based on a Boltzmann transport equation (BTE) with rates that account for all possible scattering channels $[52,53]$. However, BTE calculations are computationally demanding, in particular when different phonon modes should be described. The method we consider here is only approximate, but since the computational resources required are modest, it opens the way to an efficient exploration of the parameter space defined by the nanoparticle and pulse features. As an additional benefit, the approach is amenable to an analytical treatment, an insight that is difficult to obtain with the BTE.

The governing equations given above define a fourtemperature model for the CSNP-water system, which is a straightforward extension of the TTM [54,55]. The key point of the TTM is to describe the nonequilibrium dynamics of electrons and phonons by assuming coupled rate equations for the different energy carriers. Predictions of the TTM were partly confirmed by early experimental observations of nonequilibrium between electrons and phonons in metal with use of time-domain thermoreflectance as reported by Eesley [56]. The TTM is based on a number of standard but simplifying assumptions. Because we apply this framework for particles of size below $100 \mathrm{~nm}$ and for laser excitation of very short timescales (down to $1 \mathrm{ps}$ ), it is important to discuss the expected range of validity. We discuss in turn the three main assumptions underlying our governing equations:

(a) Fast electron thermalization

(b) Diffusive or ballistic transport of the energy carriers

(c) Bulk near-equilibrium values for the transport coefficients.

\section{Fast electron thermalization}

Electron thermalization depends on the rate of both electron-photon and electron-electron processes. The electron-photon interactions first bring the valence electrons [57] to a high-energy state [58]. The initial electron distribution is a nascent (as-photoexcited) distribution that differs from the Fermi-Dirac distribution $f_{\mathrm{FD}}\left(\epsilon, \mu\left(T_{e}\right)\right)=$ $1 / \exp \left\{\left[\epsilon-\mu\left(T_{e}\right)\right] / k_{B} T_{e}\right\}[59,60]$. Indeed, at very short timescales excited electrons are not "dressed" by electronelectron interactions and thus they may not be viewed as quasiparticles. Is it only after sufficient electron-electron collisions have occurred that the electron density may by described by a Fermi-Dirac distribution with a welldefined local electronic temperature $T_{e}$. The corresponding electron-electron scattering time $\tau_{e e}$ may be inferred from liquid Fermi theory $[61,62]$ and depends on the excess energy of the electron $\delta \epsilon=\epsilon-\epsilon_{F}$ with respect to the Fermi level $\epsilon_{F}: \tau_{e e}=\tau_{0}\left(\epsilon_{F} / \delta \epsilon\right)^{2}$ in the randomphase approximation $[59,62]$, where $\tau_{0}=128 /\left(\pi^{2} \sqrt{3} \omega_{p}\right)$, where $\omega_{p}$ is the plasma frequency. This scattering time describes the relaxation time of an electron due to its interaction with a reservoir of thermalized electrons. The thermalization time of the electrons, however, is a collective phenomenon occurring on a timescale $\tau$, which may be calculated with use of Boltzmann transport equations of the electron-electron scattering process characterized by the time $\tau_{e e}$. Monte Carlo simulations demonstrated that the relaxation time $\tau$ generally decreases with the laser fluence [63]. The sensitivity of the electron relaxation time on the laser fluence explains why early experimental investigations, at low excitation levels, reported a nonthermal character of the electron distribution [59,60,64-67]. At higher excitations, the electron cooling down is relatively well described by the two-temperature model $[63,68-70]$.

For the specific case of gold and for electron temperatures higher than $800 \mathrm{~K}$, the relaxation time computed from Monte Carlo simulations [63] is shorter than $1 \mathrm{ps,}$ and in this work we concentrate on this latter regime. For femtosecond pulses, this implies that the incident laser power is above a minimum value that is typically a few watts. In those conditions, the fast electron thermalization underlying the TTM is a reasonable approximation.

\section{Diffusive or ballistic transport of the energy carriers}

As demonstrated by first-principle calculations [71], the vast majority of phonon modes in gold have a mean free path shorter than $10 \mathrm{~nm}$ [72]. Because the gold nanostructures of interest here are much larger, the assumption of diffusive transport for gold phonons is well justified (some alternatives were proposed in Ref. [73]). In contrast, it has been shown experimentally that the electron mean free path $\delta$ in gold is $100 \mathrm{~nm}$ [74-77], which is greater than the typical size of the nanoparticle considered in this work. In view of this quasiballistic nature of the transport, we assume that the electronic temperature $T_{e}$ is uniform all across the metallic region [see Eq. (1)]. Furthermore, the spatial dependence of the gold-phonon temperature is also 
ignored [see Eq. (2)], assuming in effect a flat profile. As discussed in Appendix C, this hypothesis is numerically validated in the nanostructures investigated. One convenient implication is that the radial symmetry is conserved at all times.

For amorphous silica, molecular-dynamics simulations demonstrated that Fourier's law is valid down to the nanometer scale [78], justifying our assumption of diffusive transport in the silica shell. Similarly, heat transport in liquid water can also be modeled as diffusive. Recent molecular-dynamics simulations [79] indeed showed that, in the vicinity of a metallic nanoparticle, the local thermal conductivity of water may be described by bulk water conductivity, except in the immediate vicinity $(2 \mathrm{~nm})$ of the nanoparticle and at very short timescales $(t<5 \mathrm{ps})$.

\section{Bulk near-equilibrium transport coefficients}

Given that the gold nanoparticles considered below may have a radius as small as $20 \mathrm{~nm}$, one may wonder whether we should consider the electron-phonon coupling constant of bulk gold. Early experimental investigations aimed at determining the electron-phonon coupling constant in metallic thin films [67,80-83] were prompted by theoretical predictions of the thermal relaxation in metals by Allen [84]. In the case of nanoparticles, Arbouet et al. [85] demonstrated for diameters above $10 \mathrm{~nm}$ that the intrinsic electron-phonon interaction is well accounted for by the bulk constant. This being said, we should also discuss the fact that the electron-phonon coupling constant $G$ may change with the electronic temperature $T_{e}[49,70,73,86]$. This dependence starts to be significant at high temperatures $\left(T_{e}>5000 \mathrm{~K}\right)$ for gold, and in this study we concentrate on lower-energy excitations. For completeness, we perform TTM simulations in CSNPs taking into account the temperature dependence of both the electron-phonon coupling constant and the electronic heat capacity. As detailed in Appendix B, the results indicate that these nonlinear effects have little impact in the regime considered.

In conclusion, the TTM framework may provide a sound description of thermal transport across gold-silica nanoparticles, whose gold region has a spatial dimension larger than $10 \mathrm{~nm}$, and irradiated by laser pulses of moderately high fluence, such that the maximal electronic temperature $T_{e}$ is between 800 and $5000 \mathrm{~K}$. For femtosecond pulses, this corresponds typically to incident laser powers between 2 and $100 \mathrm{~W}$. Finally, since such a power is below the threshold for water cavitation [87], we disregard all phenomena related to mass transfer or bubble formation.

\section{ANALYTICAL APPROACH}

Here we examine by analytical means to what extent the heat-relaxation dynamics differ for a CSNP and a GNP. To do so, we consider the model defined by Eqs. (1)-(4), but for tractability, we add the following approximations: (i) heat diffusion is instantaneous, implying that the temperature is uniform within each domain and that in particular, the shell thickness does not play any role; (ii) all coefficients are constant; (iii) the heating of electrons is instantaneous; and (iv) the water remains at initial temperature $T_{0}$. The system of equations is now linear, and the solution can be obtained in terms of Laplace transforms with respect to time. Transformation back to the time domain to obtain explicit expressions for the temperature $\left\{T_{m}(t)\right\}$ is not possible analytically in the general case but it is straightforward to do so once parameters are given their numerical values. Because $c_{e}$ and $\sigma_{e s}$ both depend heavily on temperature, we consider two limiting cases, where they take a low value and a high value, respectively. We use $c_{e}(T)$ and $\sigma_{e s}(T)$, with $T$ fixed to $T_{0}$ in case A and $10 T_{0}$ in case $\mathrm{B}$.

The time evolution of temperatures is shown in Fig. 2, where we compare the results with and without the electron-silica conductance. The most-striking difference is visible at short times. With $\sigma_{e s}$, the silica-shell temperature $T_{s}$ is well above that of gold phonons $T_{p}$, and reaches a maximum before $10 \mathrm{ps}$. Without $\sigma_{e s}, T_{s}$ remains largely below $T_{p}$, and the maximum is attained at a much later time, around $100 \mathrm{ps}$. The acceleration of energy transfer in the presence of electron-silica conductance is particularly visible in the electron temperature, whose decrease is shifted to earlier time over most of the time range. Finally, with $\sigma_{e s}$ there is a time interval somewhere between 1 and $100 \mathrm{ps}$ where the silica shell has the highest temperature. Because all those features are common to cases A and $\mathrm{B}$, one can expect they are relevant in the full model as well, which is confirmed by comparison with the numerical results (see Fig. 5). Overall, this suggests that the analytical solution is able to capture at least the qualitative features.

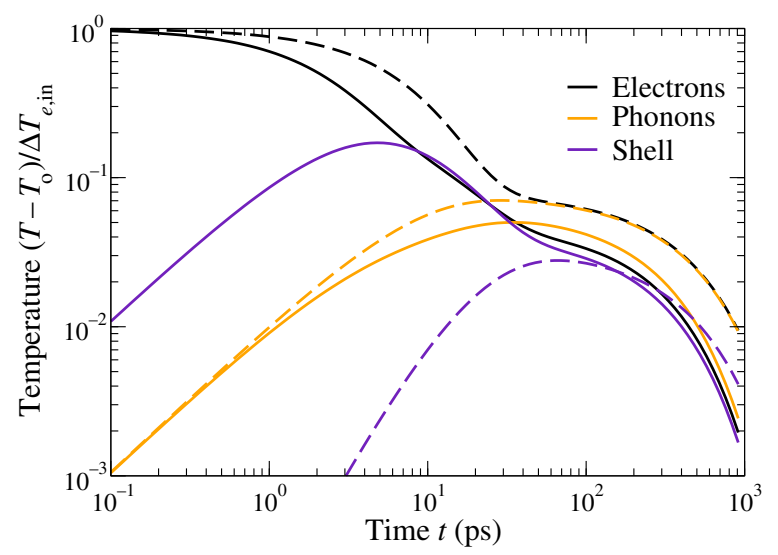

FIG. 2. Time evolution of the temperatures for the components of a CSNP with and without interfacial electron-shell conductance (continuous and dashed lines, respectively) as predicted by the analytical model. The initial shift in electron temperature is unity. 
We now characterize the short-time behavior of heat transfer. Because water is assumed to remain at temperature $T_{0}$, we consider the total heat flux going out of the particle. It increases linearly at early times:

$$
J / \Delta T_{e, \text { in }}=\kappa t+\mathcal{O}\left(t^{2}\right),
$$

where $\Delta T_{e \text {,in }}$ is the initial shift in electron temperature. The prefactor is found to be

$$
\kappa_{\mathrm{CSNP}}=\frac{12 \pi\left(R_{c}+d\right)^{2}}{R_{c}\left[\left(1+d / R_{c}\right)^{3}-1\right]} \frac{\sigma_{e s} \sigma_{s w}}{c_{s}}
$$

for the core-shell nanoparticle and

$$
\kappa_{\mathrm{GNP}}=4 \pi R^{2} \frac{\sigma_{p w} G}{c_{p}}
$$

for the gold nanoparticle. Except for the smallest particles with $R \lesssim 15 \mathrm{~nm}$, the $\kappa_{\mathrm{CSNP}} / \kappa_{\mathrm{GNP}}$ ratio computed for particles of the same total radius $R=R_{c}+d$ is approximately 2 in case A and approximately 10 in case B. The heat flux at early times is thus significantly enhanced with core-shell nanoparticles. The thin-shell limit $\epsilon=d / R_{c} \rightarrow 0$ is the most interesting and leads to a very-compact expression for the ratio,

$$
\frac{\kappa_{\mathrm{CSNP}}}{\kappa_{\mathrm{GNP}}}=\frac{c_{p} \sigma_{e s} \sigma_{s w}}{G c_{s} \sigma_{p w}}\left(\frac{1}{\epsilon}-1\right)+\mathcal{O}(\epsilon)
$$

which is independent of radius, and where the numerical prefactor is 0.38 in case A and 1.6 in case B. In the latter case, an order-of-magnitude enhancement in flux is expected for the typical value $\epsilon \simeq 0.1$, suggesting that a thin-shell CSNP is clearly to be preferred over a GNP.

One way to quantify the relaxation rate of heat transfer is to consider the time required to heat up the water in the vicinity of the particle. Accordingly, we propose an estimate for the time $\bar{\tau}$ needed to reach temperature $T_{0}+\Delta T_{w}$ in a water layer of thickness $h$. We assume that the heat released by the particle, as estimated by integration of Eq. (8), serves only to heat up homogeneously the water layer, without diffusing further, giving

$$
\frac{\bar{\tau}^{2} \Delta T_{e, \text { in }}}{2 \kappa}=\Delta T_{w} c_{w} \frac{4 \pi}{3}\left[(R+h)^{3}-R^{3}\right] .
$$

For $h=2 \mathrm{~nm}$, we get $\bar{\tau}_{\mathrm{CSNP}}=66 \sqrt{\Delta T_{w} / \Delta T_{e, \text { in }}}$ and $32 \sqrt{\Delta T_{w} / \Delta T_{e, \text { in }}}$ ps in cases $\mathrm{A}$ and $\mathrm{B}$ respectively. This line of reasoning is applicable only if the time $h^{2} / \mathcal{D}_{w}=26 \mathrm{ps}$ needed for heat diffusion in water over a distance $h$ is comparable to $\bar{\tau}_{\mathrm{CSNP}}$, which is the case as long as $\Delta T_{w} / \Delta T_{e, \text { in }}$ remains below unity.
A very-compact expression for $\bar{\tau}_{\mathrm{CSNP}}$ is obtained if we take again the thin-shell limit and expand this time at lowest order in $h / R_{c}$ :

$$
\bar{\tau}_{\mathrm{CSNP}}=\sqrt{\frac{d c_{s}}{\sigma_{e s}} \frac{h c_{w}}{\sigma_{s w}} \frac{\Delta T_{w}}{\Delta T_{e, \mathrm{in}}}},
$$

where one recognizes the geometrical average of the two relaxation times associated with interfacial conductance $\sigma_{e s}$ and $\sigma_{s w}$. Numerically, $\bar{\tau}_{\mathrm{CSNP}}=43 \sqrt{\Delta T_{w} / \Delta T_{e, \text { in }}}$ and $21 \sqrt{\Delta T_{w} / \Delta T_{e} \text {,in }}$ ps in cases $\mathrm{A}$ and $\mathrm{B}$, respectively. With $\Delta T_{e, \text { in }} \simeq 10^{3}$ and $\Delta T_{w}$ in the range from 1 to $100 \mathrm{~K}, \bar{\tau}_{\mathrm{CSNP}}$ falls in the range from 0.6 to $13 \mathrm{ps}$, which is close to the heating time found numerically below, at least for intermediate and high laser powers. In the low-power regime, near the threshold, heat presumably has time to diffuse in water [87], and Eq. (13) is not expected to apply. Finally, we can compare $\bar{\tau}_{\mathrm{CSNP}}$ for the core-shell and gold nanoparticles. From Eqs. (9), (10), and (12), one sees that the ratio $\bar{\tau}_{\mathrm{GNP}} / \bar{\tau}_{\mathrm{CSNP}}$ is controlled by $\sqrt{\kappa_{\mathrm{GNP}} / \kappa_{\mathrm{CSNP}}}$. As seen in Fig. 3, the enhancement is more pronounced for thin shells. For a typical case where $R=45 \mathrm{~nm}$ and $d=5 \mathrm{~nm}$, for cases A and $\mathrm{B}$ one gets $\bar{\tau}_{\mathrm{GNP}} / \bar{\tau}_{\mathrm{CSNP}}=1.6$ and 3.3 , respectively.

To conclude this section, our analytical approach suggests that compared with GNPs, CSNPs with thin shells may induce heat transfer that is 2 or 3 times faster. As we show in Appendix A, the presence of a silica shell may also decrease the longest relaxation time in the system. To probe the intermediate regime, which is most

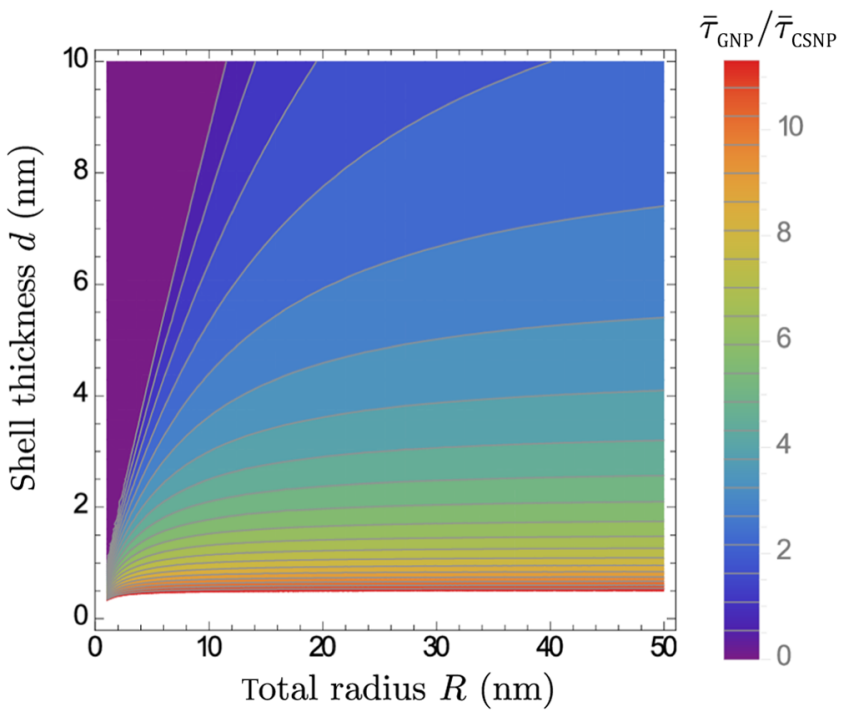

FIG. 3. Ratio of relaxation times $\bar{\tau}_{\mathrm{GNP}} / \bar{\tau}_{\mathrm{CSNP}}$ for a CSNP and a GNP as a function of the total radius $R$ and shell thickness $d$ for case B. The result for case A is quite similar, with values typically twice as small. 
relevant to applications, and obtain a complete picture, taking into account nonlinearities, we now turn to numerical resolution.

\section{NUMERICAL RESULTS AND DISCUSSION}

We present in this section our numerical results on the heat transfer around illuminated nanoparticles, with focus on the comparison between a CSNP and a GNP. The simulation method is based on a finite-difference scheme. In contrast to the analytical treatment in Sec. III, where only the short-time and long-time behaviors were accessible, we now have access to the entire relaxation process and we can assess the heating efficiency with criteria that are tailored to a specific application. For a broad range of photothermal applications, the primary goal is to heat up the immediate vicinity of the particle so as to transfer heat to the environment. This is true not only for photoacoustics [10] but also for photothermal bubble generation [88]. Therefore, we choose as our central quantity the heating time $\tau$ needed to reach a given temperature $T_{0}+\Delta T_{w}$ at a distance $h$ from the surface of the nanoparticle. Throughout the study, we fix $h=2 \mathrm{~nm}$, whereas $\Delta T_{w}$ is varied in a large range.

As a complementary measure of efficiency, we also consider the ratio

$$
\eta=\frac{E_{h}(\tau)}{E_{\text {laser }}(\tau)}
$$

where $E_{h}(t)$ is the energy stored in water within a distance $h$ from the particle surface and $E_{\text {laser }}(t)$ is the total energy supplied by the laser; both quantities are time dependent, and we consider their ratio at time $\tau$ when the desired temperature shift $\Delta T_{w}$ is reached.

Our default parameters are as follows. The initial temperature is $T_{0}=300 \mathrm{~K}$. If not otherwise mentioned, the

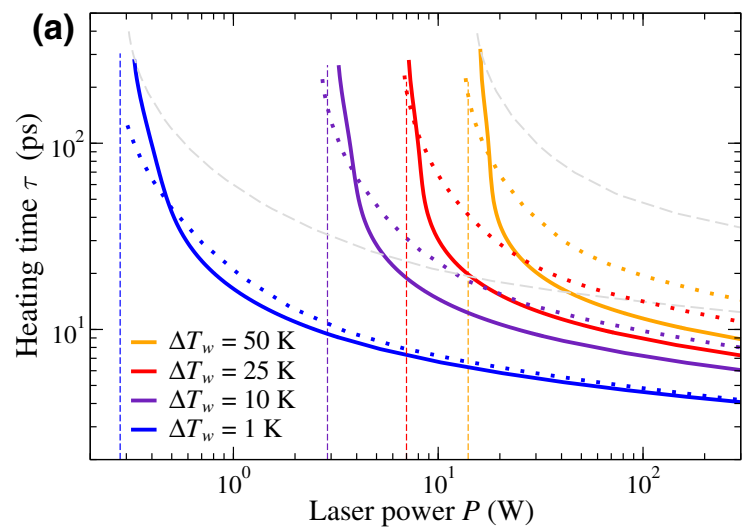

total radius of the particle is $R=45 \mathrm{~nm}$, the silica shell has thickness $d=5 \mathrm{~nm}$, and laser-pulse duration is $t_{\text {pul }}=10$ fs.

\section{A. CSNP versus GNP}

Figure 4(a) shows the heating time $\tau$ needed to reach a desired temperature shift $\Delta T_{w}$ ranging from 1 to $50 \mathrm{~K}$. We first note that $\tau$ is defined only if the laser power is above a threshold value $P_{\text {th }}$, which is proportional to the temperature shift. As can be expected, the heating time is a decreasing function of the laser power, with a steep drop in the vicinity of the threshold. Our main observation is that, away from the threshold, the CSNP heats up the water faster than the GNP. The trend is most pronounced at a high heating level. For instance, with $\Delta T_{w}=50 \mathrm{~K}$ and laser power $P=50 \mathrm{~W}$, the heating time is almost twice as short as with a CSNP. The decrease of the heating time due to the presence of a silica shell is of the same order of magnitude as reported experimentally [22]. The difference in the heating time between the two types of particles vanishes at a low heating level: for $P=50 \mathrm{~W}$, it is only $5 \%$ at $\Delta T_{w}=1 \mathrm{~K}$. This trend can be understood if we recall that the interfacial electron-silica conductance $\sigma_{e s}$ depends heavily on temperature, as specified in Eq. (7). As detailed below, the enhancement of heat transfer is thus more significant at high temperature.

Core-shell and gold nanoparticles also differ in their efficiency, as seen in Fig. 4(b). Strikingly, except for laser powers very close to the threshold, the CSNPs always perform better. For instance, with laser power $P=50 \mathrm{~W}$ and $\Delta T_{w}=1 \mathrm{~K}$, there is more than a twofold difference in efficiency, with $\eta=1.2 \%$ and $0.5 \%$ for CSNPs and GNPs, respectively. Whatever the temperature shift, the efficiency never exceeds $10 \%$ and is only a fraction of a percent at the highest power laser. In this case, most of the energy brought by the laser pulse either diffuses away or is stored

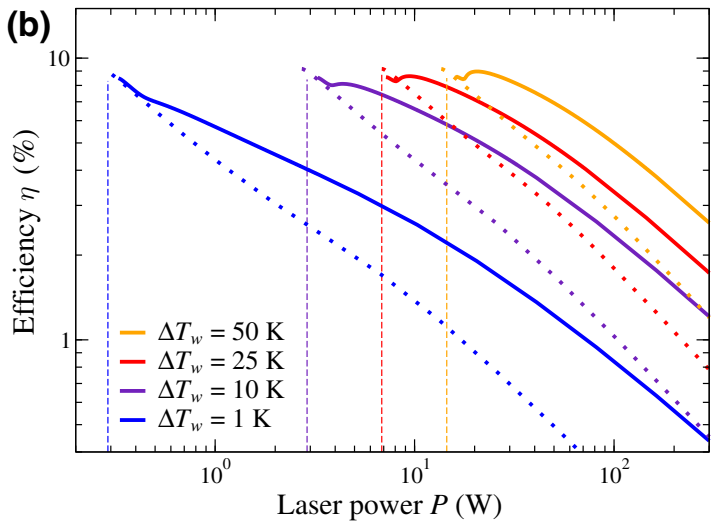

FIG. 4. Heating time $\tau$ (left) and efficiency $\eta$ (right) as a function of laser power $P$ for various temperature shifts $\Delta T_{w}$ of the water shell. Results are included for a CSNP (solid lines) and a GNP (dotted lines). In both cases, the total radius is $R=45 \mathrm{~nm}$. The dashed gray lines show the heating time for a CSNP when the electron-silica channel is disabled $\left(\sigma_{e s}=0\right)$ for $\Delta T_{w}=1 \mathrm{~K}$ (left curve) and $\Delta T_{w}=50 \mathrm{~K}$ (right curve). Vertical lines indicate the threshold power $P_{\mathrm{th}}$. 

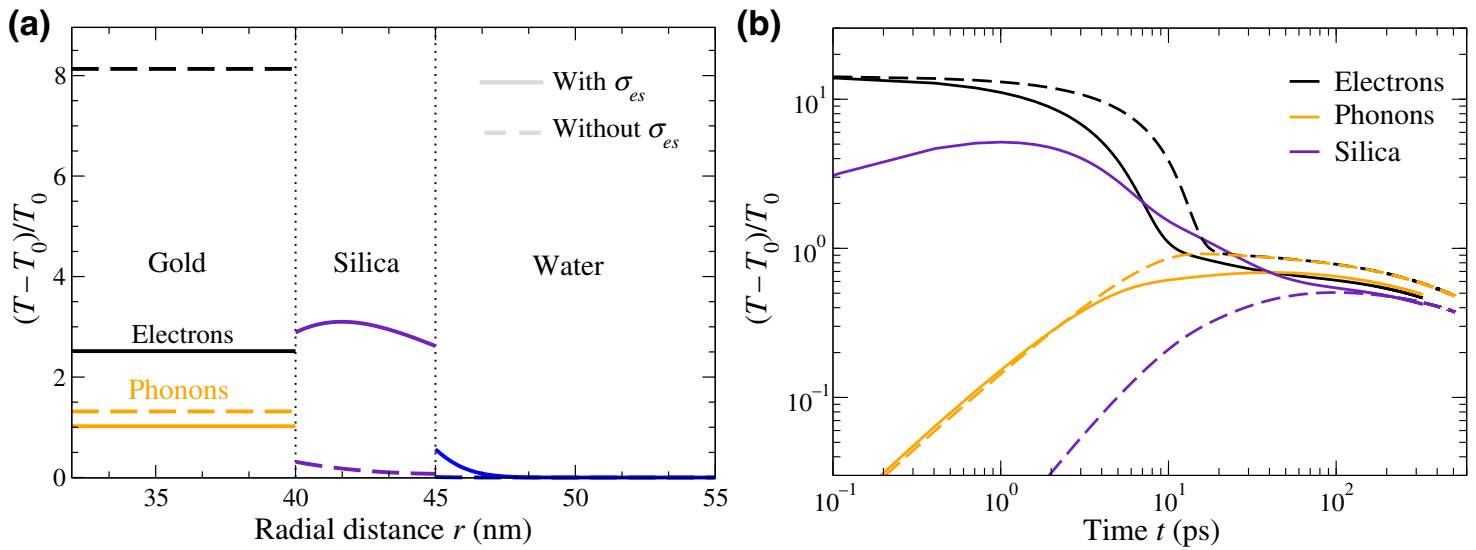

FIG. 5. Temperature within a CSNP with and without electron-silica conductance $\sigma_{e s}$ (continuous and dashed lines, respectively). (a) Local temperature for each domain at time $t=10 \mathrm{ps}$. The laser power is $P=13.9 \mathrm{~W}$, close to the threshold. (b) Time evolution of the mean temperature of each component.

in the nanoparticle. Because the heating time is minimum well above the threshold, whereas the efficiency is maximum near the threshold, an optimal value of the laser power should exist if the two criteria are to be optimized simultaneously.

\section{B. Role of the interfacial electron-silica conductance}

CSNPs may heat up the neighboring water shell faster and more efficiently than GNPs. If however, the electronsilica coupling is disabled $\left(\sigma_{e s}=0\right)$, this conclusion no longer holds, as illustrated in Fig. 4(a) by the dashed lines. The heating time will become longer for CSNPs. To better appreciate the effect of $\sigma_{e s}$ on the heating dynamics, we plot in Fig. 5 the temperature profile within the CSNP and its time evolution with and without $\sigma_{e s}$. Whereas the phonon temperature is rather insensitive to the presence of this channel, both the electron temperature and the silica temperature are strongly affected. For instance, at time $t=10$ ps shown in Fig. 5(a), the electron temperature is

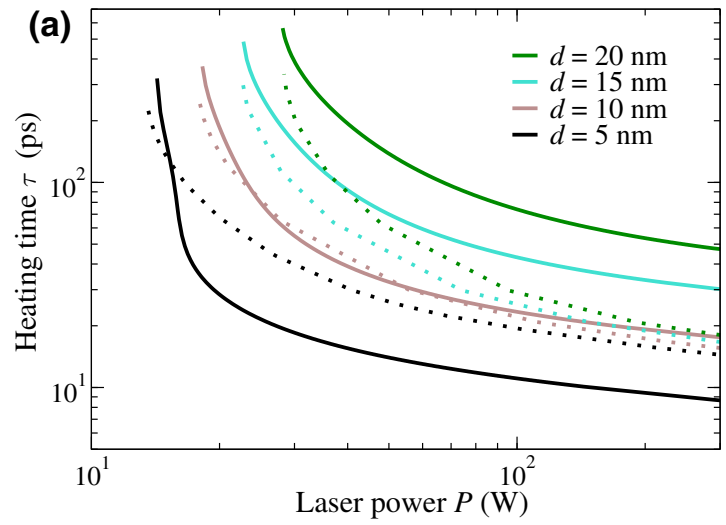

4 times lower, whereas the silica temperature is higher by an order of magnitude. Surprisingly, the silica component can be the hottest component in the system. The time evolutions of the different temperatures in the system are shown in Fig. 5(b). The electrons are the first to reach their maximum temperature at the end of the illumination by the laser power (not shown). Around a time of $1 \mathrm{ps}$, the silica in turn reaches a maximum at $T_{s} \simeq 1800 \mathrm{~K}$, well above the phonon temperature at this stage, a phenomenon already noticed with the analytical model. The picture is completely modified without $\sigma_{e s}$. The maximum silica temperature is much lower $\left(T_{s} \simeq 450 \mathrm{~K}\right)$ and occurs at a much later time $\left(10^{2} \mathrm{ps}\right)$. Thus, the acceleration of the energy transfer that was already present in the simplified analytical model is fully confirmed by the numerical results.

\section{Influence of particle size and shell thickness}

The ability of a particle to store energy is related to its geometrical and thermophysical parameters, notably the

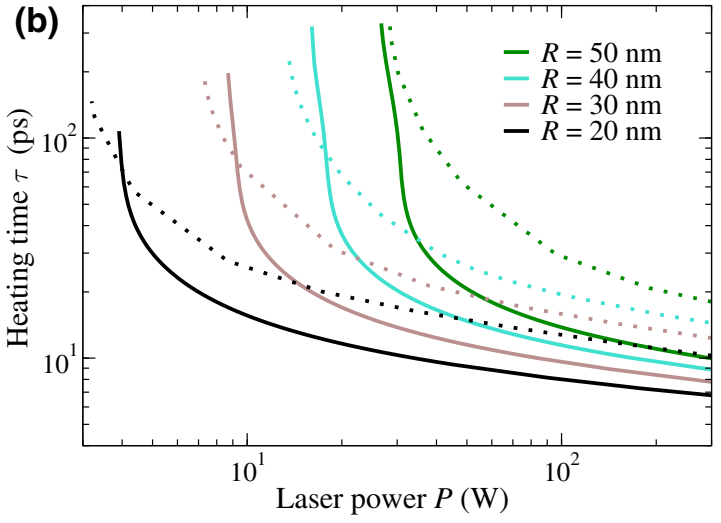

FIG. 6. Influence of geometrical properties of a CSNP on the heating time. (a) The shell thickness $d$ is varied while the core radius $R_{c}=40 \mathrm{~nm}$ is maintained. (b) The total radius $R$ is varied while the shell thickness $d=5 \mathrm{~nm}$ is maintained. The temperature shift $\Delta T_{w}=50 \mathrm{~K}$. The dotted lines indicate the results for a GNP of the same total radius. 

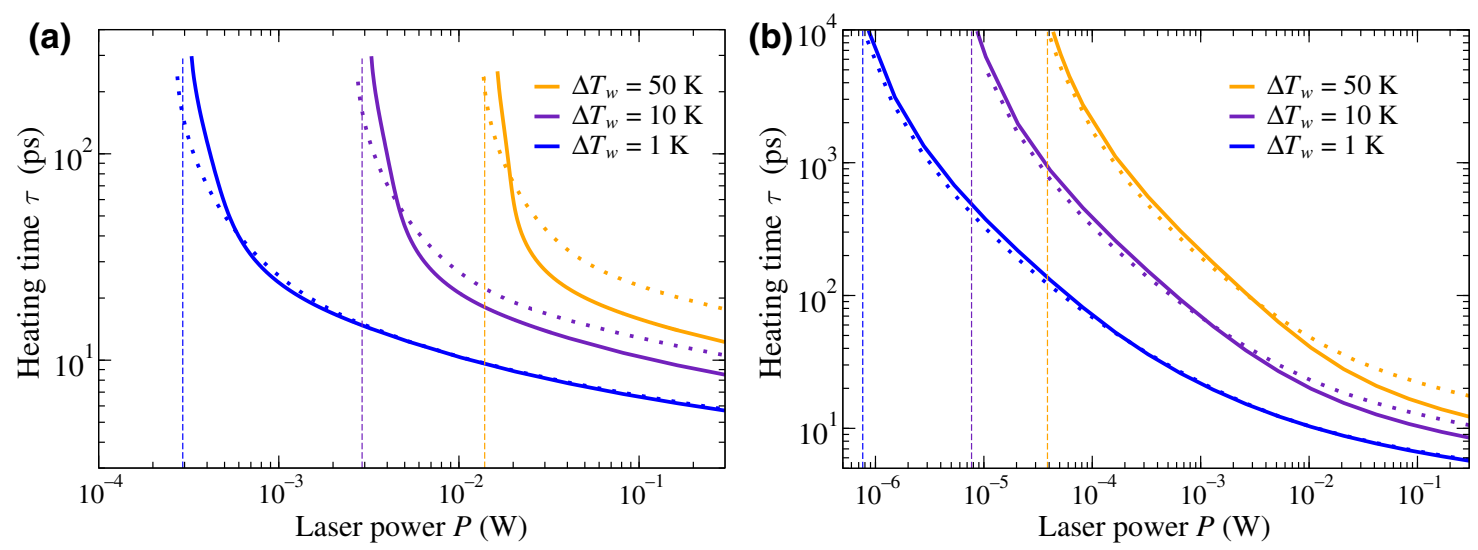

FIG. 7. Influence of the laser-pulse duration on the heating time: (a) $t_{\text {pul }}=10 \mathrm{ps}$; (b) $t_{\text {pul }}=10$ ns. The water-temperature shift is $\Delta T_{w}$. Results for a CSNP and a GNP are shown in continuous and dashed lines, respectively. Here $R_{c}=40 \mathrm{~nm}$ and $d=5 \mathrm{~nm}$.

thermal diffusivity $\mathcal{D}$ and heat capacity $c$, which are fixed once materials are chosen. On the other hand, both the size of the metallic core and the thickness of the silica shell can be tailored at will. In what follows, we investigate their influence on the energy transfer.

Figure 6 illustrates the effect of changing the shell thickness $d$. Here the gold core radius is fixed to $R_{c}=40 \mathrm{~nm}$, and the shell thickness increases from 5 to $20 \mathrm{~nm}$. The temperature shift is $\Delta T_{w}=50 \mathrm{~K}$. For comparison, the heating time for a GNP with the same total radius $R=R_{c}+d$ is also reported. A feature common to both types of particles when the size is increased is the shift in the threshold laser power, which reflects the fact that for larger particles more energy is needed to reach the same heating level in the surrounding water shell. Above the threshold, CSNPs and GNPs display different behaviors. When the laser power $P$ exceeds $100 \mathrm{~W}$, the heating time of GNPs varies only slightly with their size. In contrast, the heating time of CSNPs increases considerably with shell thickness. Such a trend can be expected since the thermal conductivity of silica is low compared with that of a metal, and heat transfer is strongly limited by heat diffusion in the silica shell (see Ref. [44] for an illustration). As a result, a CSNP with the thickest shell $d=20 \mathrm{~nm}$ yields a heating time that is almost an order of magnitude longer than that of a GNP of equal radius, whereas the thinnest shell leads to a relaxation that is still faster. It is thus clear that thin shells favor fast heat relaxation.

The core particle size can also impact the heating performance. Fixing the silica shell thickness at $d=5 \mathrm{~nm}$, we vary the core radius from 20 to $50 \mathrm{~nm}$. The heating time is shown in Fig. 6(b) and appears to decrease with the core size. It is found that small core-shell particles are beneficial in two ways. First, the heating time decreases with the nanoparticle size. Second, CSNPs perform better then GNPs due to the interfacial electron-phonon coupling. If one seeks fast heating in the water shell, the optimal nanoparticle appears to be a small CSNP with a thin shell.

\section{Influence of pulse duration}

The results so far are for a laser pulse of duration $10 \mathrm{fs}$. In this subsection, we investigate how the dynamics of heat transfer is affected by a much longer pulse, in the picosecond or nanosecond range. Although the mechanisms are the same, the heating time and threshold power exhibit drastic changes.

The heating time for pulse duration $t_{\mathrm{pul}}=10 \mathrm{ps}$ is shown in Fig. 7(a) [89]. In comparison with the short-pulse results shown in Fig. 4, the typical values for $\tau$ are similar but the threshold power decreases by 3 orders of magnitude. For instance, for $\Delta T_{w}=50 \mathrm{~K}$, we have $P_{\text {th }}=0.014 \mathrm{~W}$ rather than $14 \mathrm{~W}$ previously. This trend continues for muchlonger pulses. The heating time for laser-pulse duration $t_{\text {pul }}=10 \mathrm{~ns}$ is shown in Fig. 7(b). The threshold powers now fall in the range from $10^{-4}$ to $10^{-6} \mathrm{~W}$ depending on the temperature. Choosing again $\Delta T_{w}=50 \mathrm{~K}$, one sees that $P_{\text {th }}$ drops one more time by more than 2 orders of magnitude, now reaching $P_{\text {th }} \simeq 4 \times 10^{-5} \mathrm{~W}$. The heating time now extends over a much larger range, up to $10^{4} \mathrm{ps}$. In the vicinity of the threshold, the heating time is typically on the order of the pulse duration, implying that all the laser energy is needed before the heating criterion can be fulfilled.

A remarkable observation here is that for the longest pulse duration, the heating time of the CSNP and the GNP are almost the same in most of the laser-power range, except for the highest values $P \gtrsim 10^{-2} \mathrm{~W}$. This applies whatever the desired temperature shift. To understand this result, we first note that the typical time for the thermal energy to diffuse within the silica shell is $d^{2} / \mathcal{D}_{s} \simeq 18 \mathrm{ps}$, which is much less than the pulse duration $t_{\text {pul }}=10 \mathrm{~ns}$. Therefore, the heat transfer is not limited by equilibration 
within the silica domain. To gain further insight, we look at the temperature evolution within a CSNP and a GNP (see Fig. S2 in Ref. [44]). Except for the very early time before $1 \mathrm{ps}$, the electron, phonon, and water temperatures are identical in the two types of particles. Thus, in contrast to short pulses, for which the temperatures of the three particles are far from equilibrated, long laser pulses leave sufficient time for thermalization of the different components within the particle. The heat relaxation is then limited by the transfer to water and diffusion therein. Given that the gold-water conductance and silica-water conductance have similar values (see Table II), one can expect very similar dynamics for the CSNP and the GNP.

\section{CONCLUSION}

To summarize, we present a complete study of heat transfer around illuminated gold-silica core-shell nanoparticles immersed in water. We describe the energy transfer by analyzing a simplified model and then solving numerically the heat-transfer equations. Our model takes into account the thermal boundary conductance at the particlewater and core-shell interfaces, and highlights the role of the interfacial electron-phonon coupling. Throughout the study, we compare results for a CSNP and a GNP of the same size. To assess the heating dynamics, we chose as a quantitative criterion the time needed to increase the temperature of a thin narrow shell of water surrounding the particle. Our results show that in many situations the CSNPs enable heat transfer that is faster than that enabled by GNPs. We identify optimal conditions to minimize the heating time: a short laser pulse and a thin silica layer.

We focus exclusively on silica-coated gold nanoparticles, but it is worth discussing whether our approach and conclusions may extend to other systems. The effect that we outline here relies on the interfacial electronphonon coupling $\sigma_{e s}$. This coefficient has been measured experimentally for gold-dielectric interfaces $[23,24,90]$ but remains essentially unknown for other metals. Therefore, it would be extremely valuable to obtain from time-domain thermoreflectance or first-principles calculations, the interfacial conductance for other metals. A further question is to ascertain whether the assumptions inherent to the TTM framework still apply in the case of other systems. Several clues are already available. First, we note that gold has a relatively long electron thermalization time as compared with other metals, as shown by Boltzmann transport calculations [63]. Second, gold has a rather small electron mean free path [74]. Finally, first-principles calculations demonstrate that for gold, aluminum, and silver, the distribution of phonon mean free paths essentially vanishes above $10 \mathrm{~nm}$. Therefore, we conclude that for metals other than gold, our TTM treatment should hold under comparable and possibly broader conditions of laser heating.

Our analysis may help in interpreting two types of experimental investigations. The first involves timeresolved spectroscopy experiments [22], which showed that gold-silica core-shell nanoparticles in water display faster heat dissipation than gold nanoparticles. Second, our analysis confirms the scenario put forward in recent photoacoustic studies $[10,21]$ that interpreted the enhanced photoacoustic signal displayed by gold-silica nanoparticles as stemming from the increased efficiency of heat transfer to the environment due to the presence of a silica shell. As we show in this study, the key process here is the coupling between the metal electrons and silica phonons. If the silica shell is thin enough, the mechanism may offer optimized conditions for heat transfer from the gold core to the nanoparticle environment. The nonlinear photoacoustic response of CSNPs may be analyzed either by the analytical approach of Calasso et al. [91] in the linear regime or by solving the finite-difference time-domain numerical scheme of Prost et al. [11]. The frontier between the linear and nonlinear regimes corresponds to laser fluence $F \simeq 7 \mathrm{~mJ} / \mathrm{cm}^{2}$, which for CSNPs irradiated by femtosecond laser pulses with a wavelength in the vicinity of the surface plasmon resonance corresponds to power $\mathcal{P} \simeq 20 \mathrm{~W}$. Note that a recent study solved photoacoustic equation for silica-coated nanoparticles based on a Fourier heat-conduction model [42]. However, that work does not treat electron-phonon coupling processes, and addresses mainly long laser pulses (in the nanosecond range). In contrast, our study demonstrates that for femtosecond and picosecond laser pulses, electron-phonon couplings can not be ignored and yield an enhanced thermal response of the nanoparticle environment. This provides motivation for a full determination of the photoacoustic response of CSNPs following pulsed irradiation taking into account thermal diffusion and coupling between all the energy carriers.

This study has implications on both the applied side and the fundamental side. First, it calls for the experimental or theoretical determination of the electron-phonon conductance of different combinations of metal-dielectric interfaces. Of special interest will be interfaces displaying high thermal coupling. Enhancing locally heat transfer is important for a broad array of applications, among which photoacoustic generation may be the most-illustrative example $[10,11]$. Here, the simplest type of a core-shell nanoparticle is envisaged, but clearly interfacial electronphonon couplings should play a major role in a wide class of heterogeneous particles, including concentrated coreshell multilayers [18,92]. Combining the optical response of these structures with heat transfer mediated by the different channels opens an avenue for the optimization of photothermal energy conversion based on metal-dielectric multilayer nanostructures. 


\section{ACKNOWLEDGMENTS}

We acknowledge illuminating discussions with $\mathrm{P}$. Maioli, A. Crut, F. Amblard, and T. Niehaus. J.L. acknowledges funding from DGAPA UNAM. S.M. acknowledges financial support from the FET Open project EFINED under Grant Agreement No. 766853.

\section{APPENDIX A: LONGEST RELAXATION TIME IN THE THEORETICAL MODEL}

Because the water temperature is assumed to remain at its initial value $T_{0}$, the long-time behavior is exponential and involves a relaxation time that we characterize in this section. Skipping the derivation, one gets for the core-shell nanoparticle

$$
\begin{gathered}
\hat{\tau}_{\mathrm{CSNP}}=\frac{1}{3} \frac{G R_{c}\left(c_{e}+c_{p}\right)+3 c_{e} \sigma_{p s}}{G R_{c}\left(\sigma_{e s}+\sigma_{p s}\right)+3 \sigma_{e s} \sigma_{p s}} \\
+\frac{V_{c}\left(c_{e}+c_{p}\right)+V_{s} c_{s}}{S \sigma_{s w}},
\end{gathered}
$$

which includes the two limiting cases

$$
\begin{aligned}
\sigma_{e s} \longrightarrow \infty, \quad \hat{\tau}_{\infty} & =\frac{V_{c}\left(c_{e}+c_{p}\right)+V_{s} c_{s}}{S \sigma_{s w}}, \\
\sigma_{e s} \longrightarrow 0, & \hat{\tau}_{0}=\frac{c_{e}}{G}+\frac{R_{c}\left(c_{e}+c_{p}\right)}{3 \sigma_{p s}}+\hat{\tau}_{\infty} .
\end{aligned}
$$

Here $\hat{\tau}_{\infty}$ is the relaxation time for a core-shell nanoparticle where all components (gold electrons and phonons, silica phonons) would be equilibrated at the same temperature. $c_{e} / G$ is a typical time for equilibration between electrons and phonons. The second term on the right-hand-side of Eq. (A3) is the typical relaxation time for the ensemble of electrons and phonons, assuming they have the same temperature. To assess the effect of the electron-silica conductance, the relaxation time $\hat{\tau}_{\mathrm{CSNP}}$ is plotted as a function of $\sigma_{e s}$ in Fig. 8. Whatever the value chosen for $c_{e}$, the trend is similar. After taking a constant value at low $\sigma_{e s}$, the relaxation time decreases to another plateau, which is approximately 2 times lower. The two values considered in cases $\mathrm{A}$ and $\mathrm{B}$ fall around the upper end of the range from $10^{7}$ to $-10^{9} \mathrm{MW} \mathrm{m}^{-2} \mathrm{~K}^{-1}$ over which the drop occurs. Thus, as previously found with other criteria, one also sees in the long-time behavior that ignoring the electron-silica conductance would significantly slow down the relaxation in the system.

To compare the cases of core-shell and gold nanoparticles, we compute the longest relaxation time for the latter and find

$$
\hat{\tau}_{\mathrm{GNP}}=\frac{c_{e}}{G}+\frac{R\left(c_{e}+c_{p}\right)}{3 \sigma_{p w}},
$$

where one can recognize the sum of the electron-phonon equilibration time and the relaxation time for a bare

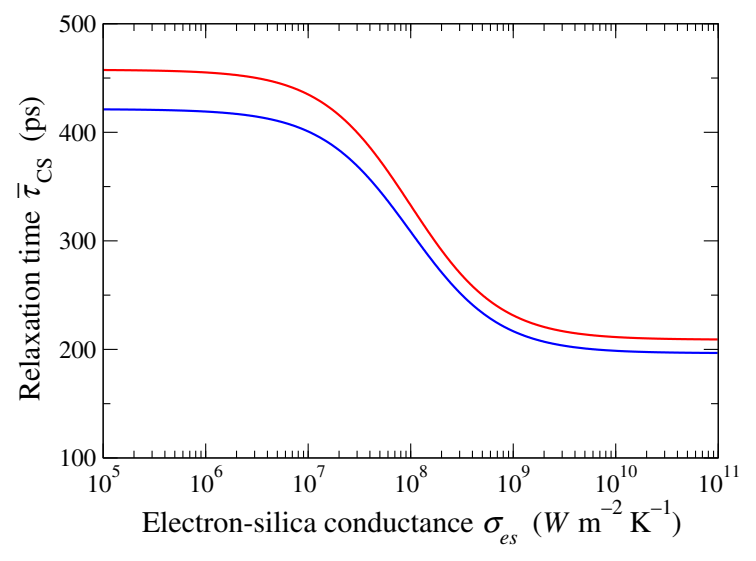

FIG. 8. Relaxation time $\hat{\tau}_{\mathrm{CSNP}}$ as a function of $\sigma_{e s}$ for $c_{e}$ fixed at $c_{e}\left(T_{0}\right)$ and $c_{e}\left(10 T_{0}\right)$ (bottom and top curves, respectively).

nanoparticle where the electron and phonon are equilibrated. This latter equation generalizes the equation ruling the heat dissipation time of a bare nanoparticle with Kapitza resistance [93], the latter time being obtained in the limit $G \rightarrow \infty$ and $c_{e} \rightarrow 0$.

Introducing the ratio of relaxation times $\xi=\hat{\tau}_{\mathrm{GNP}} / \hat{\tau}_{\mathrm{CSNP}}$ for core-shell and bare particles of the same size $R_{c}+d=$ $R$, we find that the core-shell nanoparticles are more advantageous $(\xi>1)$ for a small size and thicker silica shells. At this point, one should remember that our theoretical model assumes infinitely fast diffusion in the silica shell, implying that it is valid only for small shell thickness $d$. An ad hoc but simple way to account for diffusion is to add to $\hat{\tau}_{\text {CSNP }}$ the typical time for diffusion in the silica shell, $d^{2} / \mathcal{D}_{s}$. This is done in Fig. 9, which shows $\xi$ as a function of the total radius and shell thickness. As expected, the optimal shell thickness is now finite. For instance, it is around $6 \mathrm{~nm}$ for a particle of total radius $45 \mathrm{~nm}$. The enhancement is weaker in this case, with $\xi$ mostly less than 1.5 .

\section{APPENDIX B: EFFECT OF NONLINEAR TEMPERATURE DEPENDENCE OF THE ELECTRON-PHONON COUPLING CONSTANT $G$ AND ELECTRONIC HEAT CAPACITY $c_{e}$}

Throughout our study, we use for the electron-phonon coupling constant $G$ and the electronic heat capacity $c_{e}$ the values close to equilibrium. Under these conditions, $G$ is constant, while $c_{e}=\gamma T_{e}$ increases with the electron temperature, $\gamma$ being the Sommerfeld constant. For electronic temperatures higher than approximately $4000 \mathrm{~K}$ for gold, interband transitions start to play a role, and both $G$ and $c_{e}$ deviate from their nearequilibrium values and become nonlinear functions of $T_{e}$, as shown by first-principles $a b$ initio calculations [49]. As seen in Fig. 10, the deviations from the near-equilibrium predictions for $G$ and $c_{e}$ occur above electronic temperature $T_{e}>3000 \mathrm{~K}$. 


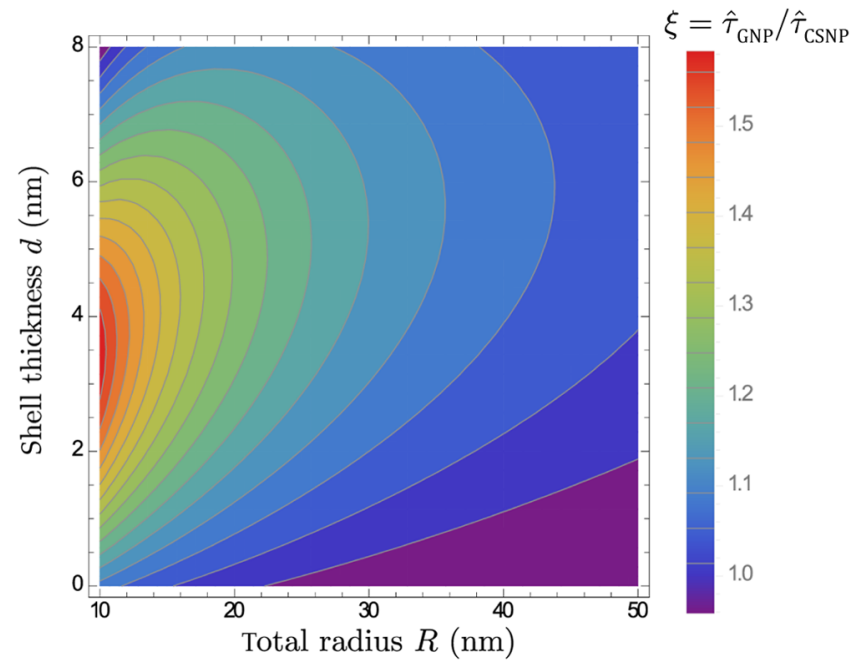

FIG. 9. Ratio of the longest relaxation time for a CSNP and a GNP as a function of total radius $R$ and shell thickness $d$ in case B. Here the diffusion in the silica shell is accounted for in a approximate manner (see the text).

To assess the effect of such nonlinearities on the CSNP heating kinetics, we show in Fig. 11 the heating time as a function of the laser power when we take into account or do not take into account the nonlinearities of both $G$ and $c_{e}$. It is clear that below $100 \mathrm{~W}$, the nonlinearities do not play a significant role, and the linear model used above provides an acceptable description of the heating of the CSNP. Beyond $100 \mathrm{~W}$, a departure from the linear model may be seen, but it should be noted that when $\sigma_{e s} \neq 0$, the deviations are limited to $15 \%$. It should also be noted that above $100 \mathrm{~W}$ we use a value of $\sigma_{e s}$ extrapolated from its low- $T_{e}$ behavior, as we have no information on
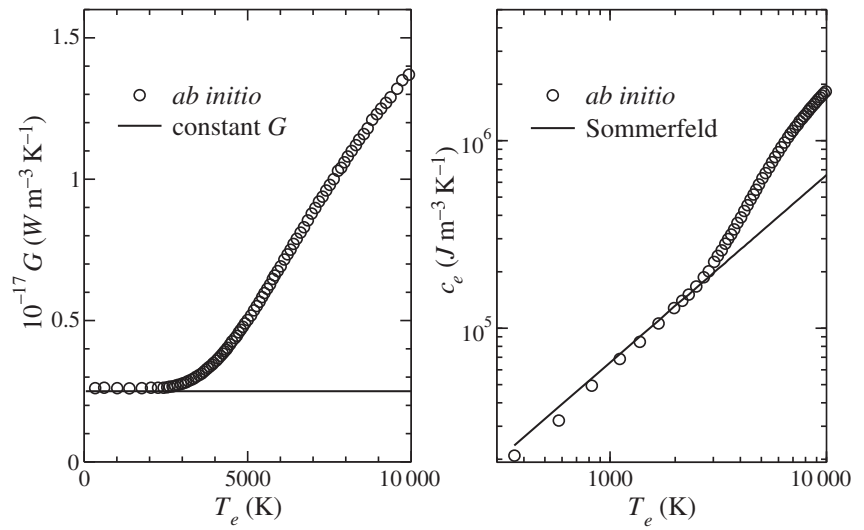

FIG. 10. Electron-phonon coupling $G$ (left) and electronic heat capacity $c_{e}$ (right) of bulk gold as a function of the electronic temperature. The results of first-principles ab initio calculations (circles) are compared with the near-equilibrium coupling constant and the Sommerfeld heat capacity (lines).

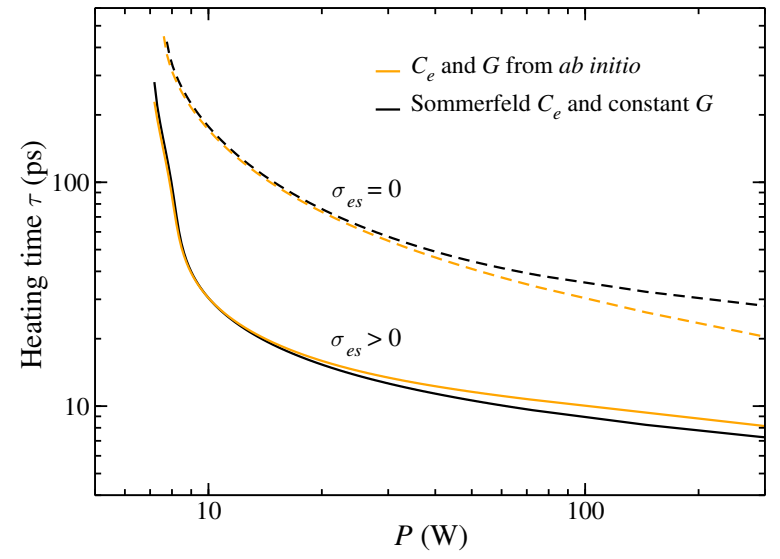

FIG. 11. Heating time to reach $\Delta T_{w}=25 \mathrm{~K}$ in two cases: constant $G$ and Sommerfeld heat capacity $c_{e}$ (black) versus ab initio values (orange). Dashed curves correspond to the case $\sigma_{e s}=0$, while continuous curves are the results with nonzero $\sigma_{e s}$. The nanoparticle considered here is a CSNP with $R_{c}=40 \mathrm{~nm}$ and $d=5 \mathrm{~nm}$ irradiated by a femtosecond laser pulse.

the electron-phonon cross-coupling at electronic temperatures $T_{e}>3000 \mathrm{~K}$. Exploring the heating kinetics of the CSNP in this regime would require the experimental determination of $\sigma_{e s}$ under conditions of strong electron-phonon nonequilibrium.

\section{APPENDIX C: EFFECT OF FINITE DIFFUSION IN THE METAL}

The model described by Eqs. (1)-(4) assumes that the diffusion of heat carriers in the metal is infinitely fast. The underlying reason is the high thermal conductivity of the gold free electrons (see Table I), suggesting that the temperatures in the metal can be approximated as uniform. While this is a good approximation for electrons, it might

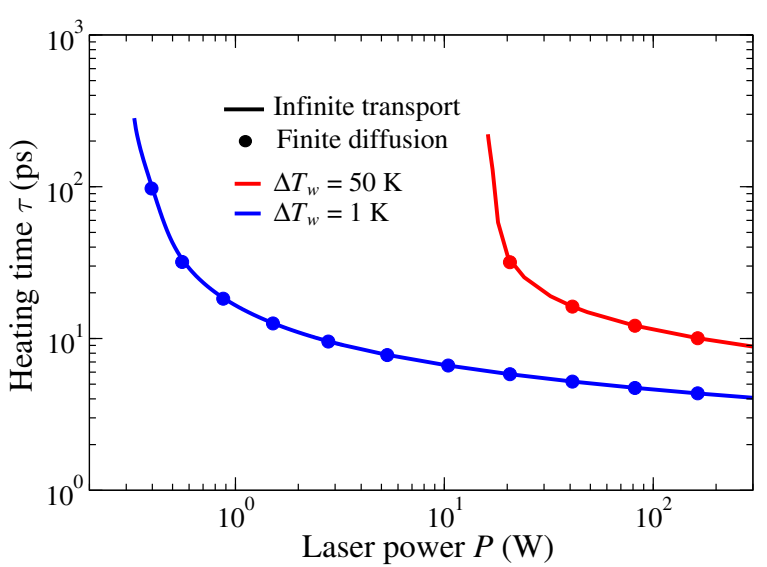

FIG. 12. Heating time to reach $\Delta T_{w}=1$ and $50 \mathrm{~K}$ assuming for metal phonons instantaneous transport (lines) or finite diffusion (circles). The nanoparticle considered here is a CSNP with $R_{c}=$ $40 \mathrm{~nm}$ and $d=5 \mathrm{~nm}$. 
not be the case for phonons, since their thermal diffusivity $\mathcal{D}_{p}$ is much smaller. Their diffusion time over a typical length of $40 \mathrm{~nm}$ is $1250 \mathrm{ps}$, which is 2 orders of magnitude greater than that for electrons. We check how heat transfers are affected when the diffusion of phonons in the metal is finite. As demonstrated in Fig. 12, the heating times are exactly the same with infinite transport or finite diffusion of phonons inside the metal. This indicates that our approximation is legitimate.

[1] C. Bohren and D. Huffman, Absorption and Scattering of Light by Small Particles (Wiley, New York, 1983).

[2] A. O. Govorov and H. H. Richardson, Generating heat with metal nanoparticles, Nano Today 2, 30 (2007).

[3] G. Baffou and H. Rigneault, Femtosecond-pulsed optical heating of gold nanoparticles, Phys. Rev. B 84, 035415 (2011).

[4] G. Baffou and R. Quidant, Thermo-plasmonics: Using metallic nanostructures as nano-sources of heat, Laser Photonics Rev. 7, 171 (2013).

[5] C. Burda, X. Chen, R. Narayanan, and M. El-Sayed, Chemistry and properties of nanocrystals of different shapes, Chem. Rev. 105, 1025 (2005).

[6] H. H. Richardson, Z. N. Hickman, A. O. Govorov, A. C. Thomas, W. Zhang, and M. E. Kordesch, Thermooptical properties of gold nanoparticles embedded in ice: Characterization of heat generation and melting, Nano Lett. 6, 783 (2006).

[7] L. Cao, D. N. Barsic, A. R. Guichard, and M. L. Brongersma, Plasmon-assisted local temperature control to pattern individual semiconductor nanowires and carbon nanotubes, Nano Lett. 7, 3523 (2007).

[8] E. C. Dreaden, A. M. Alkilany, X. Huang, C. J. Murphy, and M. A. El-Sayed, The golden age: Gold nanoparticles for biomedicine, Chem. Soc. Rev. 41, 2740 (2012).

[9] Z. Qin and J. C. Bischof, Thermophysical and biological responses of gold nanoparticle laser heating, Chem. Soc. Rev. 41, 1191 (2012).

[10] Y.-S. Chen, W. Frey, S. Kim, P. Kruizinga, K. Homan, and S. Emelianov, Silica-coated gold nanorods as photoacoustic signal nanoamplifiers, Nano Lett. 11, 348 (2011).

[11] A. Prost, F. Poisson, and E. Bossy, Photoacoustic generation by a gold nanosphere: From linear to nonlinear thermoelastics in the long-pulse illumination regime, Phys. Rev. B 92, 115450 (2015).

[12] G. Han, P. Ghosh, M. De, and V. M. Rotello, Drug and gene delivery using gold nanoparticles, NanoBiotechnology $\mathbf{3}$, 40 (2007).

[13] S. E. McNeil, Characterization of Nanoparticles Intended for Drug Delivery (Springer, London, 2011), Vol. 697.

[14] E. S. Day, J. G. Morton, and J. L. West, Nanoparticles for thermal cancer therapy, J. Biomech. Eng. 131, 074001 (2009).

[15] F. Alexis, E. M. Pridgen, R. Langer, and O. C. Farokhzad, Nanoparticle Technologies for Cancer Therapy (Springer, Berlin, 2010), p. 55.
[16] S. Lal, S. E. Clare, and N. J. Halas, Nanoshell-enabled photothermal cancer therapy: Impending clinical impact, Acc. Chem. Res. 41, 1842 (2008).

[17] L. R. Hirsch, R. J. Stafford, J. Bankson, S. R. Sershen, B. Rivera, R. Price, J. D. Hazle, N. J. Halas, and J. L. West, Nanoshell-mediated near-infrared thermal therapy of tumors under magnetic resonance guidance, Proc. Natl. Acad. Sci. 100, 13549 (2003).

[18] L. Meng, R. Yu, M. Qiu, and F. Javier Garcia de Abajo, Plasmonic nano-oven by concatenation of multishell photothermal enhancement, ACS Nano 11, 7915 (2017).

[19] H. Xu, Multilayered metal core-shell nanostructures for inducing a large and tunable local optical field, Phys. Rev. B 72, 073405 (2005).

[20] O. Neumann, A. Urban, J. Day, S. Lal, P. Nordlander, and N. J. Halas, Solar vapor generation enabled by nanoparticles, ACS Nano 7, 42 (2012).

[21] Y.-S. Chen, W. Frey, S. Aglyamov, and S. Emelianov, Environment-dependent generation of photoacoustic waves from plasmonic nanoparticles, Small 8, 47 (2012).

[22] M. Hu, X. Wang, G. Hartland, V. Salgueirino-Maceira, and L. Liz-Marzan, Heat dissipation in gold-silica core-shell nanoparticles, Chem. Phys. Lett. 372, 767 (2003).

[23] P. E. Hopkins, J. L. Kassebaum, and P. M. Norris, Effects of electron scattering at metal-nonmetal interfaces on electron-phonon equilibration in gold films, J. Appl. Phys. 105, 023710 (2009).

[24] L. Guo, S. L. Hodson, T. S. Fischer, and X. Xu, Heat transfer across metal-dielectric interfaces during ultrafast-laser heating, J. Heat Transfer 134, 042402 (2012).

[25] A. Majumdar and P. Reddy, Role of electron-phonon coupling in thermal conductance of metal-nonmetal interfaces, App. Phys. Lett. 84, 4768 (2004).

[26] R. E. Jones, J. C. Duda, X. W. Zhou, C. J. Kimmer, and P. E. Hopkins, Investigation of size and electronic effects on Kapitza conductance with non-equilibrium molecular dynamics, App. Phys. Lett. 102, 183119 (2013).

[27] P. E. Hopkins, Thermal transport across solid interfaces with nanoscale imperfections: Effects of roughness, disorder, dislocations, and bonding on thermal boundary conductance, ISRN Mech. Eng. 2013, 682586 (2013).

[28] Y. Wang, X. Ruan, and A. K. Roy, Two-temperature nonequilibrium molecular dynamics simulation of thermal transport across metal-nonmetal interfaces, Phys. Rev. B 85, 205311 (2012).

[29] P. Singh, M. Seong, and S. Sinha, Detailed consideration of the electron-phonon thermal conductance at metaldielectric interfaces, Appl. Phys. Lett. 102, 181906 (2013).

[30] M. L. Huberman and A. W. Overhauser, Electronic Kapitza conductance at a diamond- $\mathrm{Pb}$ interface, Phys. Rev. B 50, 2865 (1994).

[31] A. Sergeev, Electronic Kapitza conductance due to inelastic electron-boundary scattering, Phys. Rev. B 58, R10199 (1998).

[32] G. Mahan, Kapitza thermal resistance between a metal and a nonmetal, Phys. Rev. B 79, 075408 (2009).

[33] P. Zhangh, J. T. Lu, J. S. Wang, and B. Li, Thermal transport across metal-insulator interface via electronphonon interaction, J. Phys. Condens. Matter 25, 445801 (2013). 
[34] Z. Lu, Y. Wang, and X. Ruan, The critical particle size for enhancing thermal conductivity in metal nanoparticlepolymer composites, J. Appl. Phys. 123, 074302 (2018).

[35] J. Lombard, F. Detcheverry, and S. Merabia, Influence of the electron-phonon interfacial conductance on the thermal transport at metal/dielectric interfaces, J. Phys. Condens. Matter 27, 015007 (2015).

[36] Z. Lu, Y. Wang, and X. Ruan, Metal/dielectric thermal interfacial transport considering cross-interface electronphonon coupling: Theory, two-temperature molecular dynamics, and thermal circuit, Phys. Rev. B 93, 064302 (2016).

[37] A. Plech, V. Kotaidis, S. Grésillon, C. Dahmen, and G. von Plessen, Laser-induced heating and melting of gold nanoparticles studied by time-resolved x-ray scattering, Phys. Rev. B 70, 195423 (2004).

[38] V. Juvé, M. Scardamaglia, P. Maioli, A. Crut, S. Merabia, L. Joly, N. Del Fatti, and F. Vallée, Cooling dynamics and thermal interface resistance of glass-embedded metal nanoparticles, Phys. Rev. B 80, 195406 (2009).

[39] S. Hashimoto, D. Werner, and T. Uwada, Studies on the interaction of pulsed lasers with plasmonic gold nanoparticles toward light manipulation, heat management, and nanofabrication, J. Photochem. Photobio. C 13, 28 (2012).

[40] M. Chen, Y. He, and J. Zhu, Quantifying and comparing the near-field enhancement, photothermal conversion, and local heating performance of plasmonic $\mathrm{SiO}_{2} @ \mathrm{Au}$ core-shell nanoparticles, Plasmonics 14, 1019 (2018).

[41] D. Kumar, D. Gahi, and R. Soni, Simulation studies of photoacoustic response from gold-silica core-shell nanoparticles, Plasmonics 13, 1833 (2018).

[42] K. Shahbazi, W. Frey, Y.-S. Chen, S. Aglyamov, and S. Emelianov, Photoacoustics of core-shell nanospheres using comprehensive modeling and analytical solution approach, Comm. Phys. 119, 2 (2019).

[43] In the order electrons, metallic phonons, shell phonons, and water.

[44] See Supplemental Material at http://link.aps.org/supple mental/10.1103/PhysRevApplied.13.034036 for the equations obeyed for a homogeneous gold nanoparticle, the effect of the shell thickness on water-temperature dynamics, and the temperature evolution of the different energy carriers for long pulse durations].

[45] Z. Ge, D. G. Cahill, and P. V. Braun, Thermal Conductance of Hydrophilic and Hydrophobic Interfaces, Phys. Rev. Lett. 96, 186101 (2006).

[46] N. Shenogina, R. Godawat, P. Keblinski, and S. Garde, How Wetting and Adhesion Affect Thermal Conductance of a Range of Hydrophobic to Hydrophilic Aqueous Interfaces, Phys. Rev. Lett. 102, 156101 (2009).

[47] S. Merabia, J. Lombard, and A. Alkurdi, Importance of viscoelastic and interface bonding effects in the thermal boundary conductance of solid-water interfaces, Int. J. Heat Mass Transfer 100, 287 (2016).

[48] C. Kittel, Introduction to Solid State Physics (John Wiley \& Sons, New York, 1986).

[49] Z. Lin, L. V. Zhigilei, and V. Celli, Electron-phonon coupling and electron heat capacity of metals under conditions of strong electron-phonon nonequilibrium, Phys. Rev. B 77, 075133 (2008).
[50] M. Hu, J. V. Goicochea, B. Michel, and D. Poulikakos, Water nanoconfinement induced thermal enhancement at hydrophilic quartz interfaces, Nano Lett. 10, 279 (2009).

[51] S. Merabia, S. Shenogin, L. Joly, P. Keblinski, and J.-L. Barrat, Heat transfer from nanoparticles: A corresponding state analysis, Proc. Nat. Acad. Sci. 106, 15113 (2009).

[52] T. Q. Qiu and C. L. Tien, Heat transfer mechanisms during short-pulse laser heating of metals, J. Heat Transfer 115, 835 (1993).

[53] Y. Wang, Z. Lu, A. K. Roy, and X. Ruan, Effect of interlayer on interfacial thermal transport and hot electron cooling in metal-dielectric systems: An electron-phonon coupling perspective, J. Appl. Phys. 119, 065103 (2016).

[54] M. I. Kaganov, I. M. Lifshitz, and L. V. Tanatarov, Relaxation between the electrons and the crystalline lattice, Sov. Phys. JETP 39, 375 (1957).

[55] S. I. Anisimov, B. L. Kapeliovich, and T. L. Perelman, Electron emission from metal surfaces exposed to ultrashort laser pulses, Sov. Phys. JETP 39, 375 (1974).

[56] G. L. Eesley, Observation of Nonequilibrium Electron Heating in Copper, Phys. Rev. Lett. 51, 2140 (1983).

[57] Six seconds in the case of gold.

[58] A. Arbouet, Réponse optique ultrarapide de nano-objets ultrarapides: Effets de taille. Thesis, Université de Bordeaux, 2004.

[59] W. S. Fann, R. Storz, H. W. K. Tom, and J. Bokor, Direct Measurement of Nonequilibrium Electron-Energy Distributions in Subpicosecond Laser-Heated Gold Films, Phys. Rev. Lett. 68, 2834 (1992).

[60] W. S. Fann, R. Storz, H. W. K. Tom, and J. Bokor, Electron thermalization in gold, Phys. Rev. B 46, 13592 (1992).

[61] N. W. Ashcroft and N. D. Mermin, Solid State Physics (Holt, Rinehart and Winston, New York, 1976).

[62] D. Pines and P. Noziéres, The Theory of Quantum Liquids (Benjamin, New York, 1966).

[63] B. Y. Mueller and B. Rethfeld, Relaxation dynamics in laser-excited metals under nonequilibrium conditions, Phys. Rev. B 87, 035139 (2013).

[64] G. Tas and H. J. Maris, Electron diffusion in metals studied by picosecond ultrasonics, Phys. Rev. B 49, 15046 (1994).

[65] C. K. Sun, F. Vallée, L. Acioli, E. P. Ippen, and J. G. Fujimoto, Femtosecond investigation of electron thermalization in gold, Phys. Rev. B 48, 12365 (1993).

[66] R. H. Groeneveld, R. Sprik, and A. Lagendijk, Femtosecond spectroscopy of electron-electron and electron-phonon energy relaxation in Ag and Au, Phys. Rev. B 51, 11433 (1995).

[67] R. W. Schoenlein, W. Z. Lin, J. G. Fujimoto, and G. L. Eesley, Femtosecond Studies of Nonequilibrium Electronic Processes in Metals, Phys. Rev. Lett. 58, 1680 (1987).

[68] X. Y. Wang, D. M. Riffe, Y.-S. Lee, and M. Downer, Timeresolved electron-temperature measurement in a highly excited gold target using femtosecond thermionic emission, Phys. Rev. B 50, 8016 (1994).

[69] X. Y. Wang and D. M. Cahill, Limits to Thermal Transport in Nanoscale Metal Bilayers due to Weak Electron-Phonon Coupling in $\mathrm{Au}$ and $\mathrm{Cu}$, Phys. Rev. Lett. 109, 175503 (2012).

[70] A. Giri, J. T. Gaskins, B. M. Foley, R. Cheaito, and P. E. Hopkins, Experimental evidence of excited electron 
number density and temperature effects on electron-phonon coupling in gold films, J. Appl. Phys. 117, 044305 (2015).

[71] A. Jain and A. J. H. McGaughey, Thermal transport by phonons and electrons in aluminium, silver, and gold from first principles, Phys. Rev. B 93, 081206(R) (2016).

[72] The phonon mean free path we refer to here originates in phonon-phonon scattering processes.

[73] J. Chen, W. P. Latham, and J. E. Beraun, The role of electron-phonon coupling in ultrafast laser heating, J. Laser Appl. 17, 63 (2005).

[74] T. Q. Qiu and C. L. Tien, Size effects on nonequilibrium laser heating of metal films, Trans. ASME 115, 842 (1993).

[75] J. Hohlfeld, S.-S. Wellershoff, J. Güdde, U. Conrad, V. Jähnke, and E. Matthias, Electron and lattice dynamics following optical excitation of metals, Chem. Phys. 251, 237 (2000).

[76] M. Bonn, D. N. Denzler, S. Funk, M. Wolf, S.-S. Wellershoff, and J. Hohlfeld, Ultrafast electron dynamics at metal surfaces: Competition between electron-phonon coupling and hot-electron transport, Phys. Rev. B 61, 1101 (2000).

[77] M. Lejman, V. Shalagatskyi, O. Kovalenko, T. Pezeril, V. V. Temnov, and P. Ruello, Ultrafast optical detection of coherent acoustic phonons emission driven by superdiffusive hot electrons, J. Opt. Soc. Am. B 31, 282 (2014).

[78] J. M. Larkin and A. J. H. McGaughey, Thermal conductivity accumulation in amorphous silica and amorphous silicon, Phys. Rev. B 89, 144303 (2014).

[79] A. Rajabpour, S. Roham, S. Arabha, M. M. Heyhat, S. Merabia, and A. Hassanali, Thermal transport at a nanoparticlewater interface: A molecular dynamics and continuum modeling study, J. Chem. Phys. 150, 114701 (2019).

[80] S. D. Brorson, A. Kazeroonian, J. S. Moodera, D. W. Face, T. K. Cheng, E. P. Ippen, M. S. Dresselhaus, and G. Dresselhaus, Femtosecond Room-Temperature Measurement of the Electron-Phonon Coupling Constant Lambda in Metallic Superconductors, Phys. Rev. Lett. 64, 2172 (1990).

[81] H. E. Elsayed-Ali, T. Juhasz, G. O. Smith, and W. E. Bron, Femtosecond thermoreflectivity and thermotransmissivity of polycrystalline and signel-crystalline gold films, Phys. Rev. B 43, 4488 (1991).

[82] H. E. Elsayed-Ali, T. B. Norris, M. A. Pessot, and G. A. Mourou, Time-Resolved Observation of Electron-Phonon Relaxation in Copper, Phys. Rev. Lett. 58, 1212 (1987).
[83] P. M. Norris, A. P. Caffrey, R. J. Stevens, J. M. Klopf, J. T. McLeskey, Jr., and A. N. Smith, Femtosecond pump-probe nondestructive examination of materials, Rev. Sci. Instr. 74, 400 (2003).

[84] P. B. Allen, Theory of Thermal Relaxation of Electrons in Metals, Phys. Rev. Lett. 59, 1460 (1987).

[85] A. Arbouet, C. Voisin, D. Christofilos, P. langot, N. D. Fatti, F. Vallée, J. Lermé, G. Celep, E. Cottancin, M. Gaudry, M. Pellarin, M. Broyer, M. Maillard, M. P. Pileni, and M. Treguer, Electron-Phonon Scattering in Metal Clusters, Phys. Rev. Lett. 90, 177401 (2003).

[86] A. Giri and P. E. Hopkins, Transient thermal and nonthermal electron and phonon relaxation after short-pulsed laser heating of metals, J. Appl. Phys. 118, 215101 (2015).

[87] J. Lombard, T. Biben, and S. Merabia, Threshold for vapor nanobubble generation around plasmonic nanoparticles, J. Phys. Chem. C 121, 15402 (2017).

[88] J. Lombard, T. Biben, and S. Merabia, Kinetics of Nanobubble Generation around Overheated Nanoparticles, Phys. Rev. Lett. 112, 105701 (2014).

[89] For pulses of duration $10 \mathrm{ps}$ and $10 \mathrm{~ns}$, the maximal electron temperature remains below $5000 \mathrm{~K}$ as long as the laser power is below $10^{-} 1 \mathrm{~W}$. Besides, from extrapolation of Fig. 11 in Ref. [63], the electron thermalization time in gold at $300 \mathrm{~K}$ is around $5 \mathrm{ps}$, which does not exceed the pulse duration. The fast thermalization assumption is thus acceptable.

[90] A. Giri, J. T. Gaskin, B. Donovan, C. Szwejkowski, R. J. Warzoha, M. A. Rodriguez, J. Ihlefeld, and P. E. Hopkins, Mechanisms of nonequilibrium electron-phonon coupling and thermal conductance at interfaces, J. Appl. Phys. 117, 105105 (2015).

[91] I. G. Calasso, W. Craig, and G. J. Diebold, Photoacoustic Point Source, Phys. Rev. Lett. 86, 3550 (2001).

[92] R. Bardhan, S. Mukherjee, N. A. Mirin, S. D. Levit, P. Nordlander, and N. J. Halas, Nanosphere-in-a-nanoshell: A simple nanomatryushka, J. Phys. Chem. C 114, 7378 (2010).

[93] O. M. Wilson, X. Hu, D. G. Cahill, and P. V. Braun, Colloidal metal particles as probes of nanoscale thermal transport in fluids, Phys. Rev. B 66, 224301 (2002). 